\title{
A Theoretical Analysis of Season Length Restrictions in Fisheries Management*
}

\author{
QING XU ${ }^{\mathrm{a}}$ and AMITRAJEET A. BATABYAL ${ }^{\mathrm{b}} \uparrow$ \\ ${ }^{\mathrm{a}}$ American Express Cards, 4315 South 2700 West, MC 02-03-07B, Salt Lake City, UT 84184, USA; ${ }^{\mathrm{b}}$ Department of Economics, Rochester Institute of \\ Technology, 92 Lomb Memorial Drive, Rochester, NY 14623-5604, USA
}

(Received 29 September 2001)

\begin{abstract}
This paper studies season length restrictions in fisheries management from an ecological-economic perspective. We first construct a model of a stylized fishery in which season length restrictions are used to manage the fishery. We then show how the dynamic and the stochastic properties of this fishery can be used to construct two managerial criteria that are meaningful from an ecological standpoint. Finally, using these two criteria, we discuss a probabilistic approach to fisheries management in which the principal focus of a manager is on moving the fishery away from the least desirable state of existence.
\end{abstract}

Keywords: Ecological-economics; Fisheries management; Season length restrictions; Economic perspective

JEL Classification: Q22; D81

\section{INTRODUCTION}

The Atlantic surf clam fishery, the Alaskan king crab fishery, and the Pacific halibut fishery are three examples of fisheries in which season length restrictions have been frequently used to manage the fishery. More generally, as Weninger and Strand (1998) and others have noted, commercial fisheries in Canada, the USA, and in western Europe are subject to a variety of season length restrictions. $\neq$ In addition to this, in virtually every state in the USA, sport fishing seasons exist for a whole host of fish species. This tells us that the use of season length restrictions for regulatory purposes is widespread in fisheries management.

Although season length restrictions have been and are commonly used in fisheries management, there are very few theoretical studies of season length restrictions from an integrated ecological-economic perspective. Economists have studied season length restrictions in considerable detail." However, the objective of these studies has generally been to demonstrate the economic inefficiencies of season length restrictions. Karpoff (1987) is representative. After analyzing season length restrictions at some length, he concludes that such restrictions "convey distributional advantages to politically dominant fishermen at the expense of their more efficient competitors" (Karpoff, 1987, p. 192). Rarely have these economics papers studied the ecological aspects associated with the use of season length restrictions in fisheries management.

Similarly, although there are many biological studies of fisheries and of fisheries management, ${ }^{\S}$ these studies have rarely analyzed season length restrictions per se, or the economic effects of such restrictions. Given this state of affairs, this paper has three objectives. First, we construct a model of a stylized fishery in which season length restrictions are used to manage the fishery. This model accounts for the ecological and the economic aspects of the evolution of the fishery. Next, we derive two managerial criteria that are meaningful from an ecological standpoint. A distinguishing feature of our approach is that

\footnotetext{
*This paper is based on Essay 3 in the first author's doctoral dissertation in economics at Utah State University.

${ }^{\dagger}$ Corresponding author. E-mail: aabgsh@ rit.edu.

${ }^{\ddagger}$ For more on this see Karpoff (1985; 1987), Matulich et al. (1996) and Hartwick and Olewiler (1998, pp. 152-175).

${ }^{ף}$ Examples of such studies include Karpoff $(1985 ; 1987)$, Homans and Wilen (1997) and Weninger and Strand (1998).

${ }^{\S}$ See Gulland (1988), Richards and Rago (1999) and Williams (1999).
} 
these two criteria are quite unlike the managerial criteria found in traditional economic analyses of fishery management problems in which the manager maximizes some kind of welfare function. The two criteria of this paper are probabilities that are derived from the dynamic and the stochastic properties of the fishery. Finally, using these two criteria, we discuss an ecological-economic approach to the fishery management problem. In this approach, the principal focus of the manager is on moving the fishery away from the least desirable state of existence.

The paper that is most closely related to our paper is the one by Batabyal and Beladi (2001). Like this paper, Batabyal and Beladi (2001) also provide an ecologicaleconomic perspective on time restrictions in natural resource management. However, the specific methods of analysis and the objectives of these two papers are very different. In particular, unlike the objectives of this paper, Batabyal and Beladi (2001) are primarily interested in determining the likelihood of resource collapse when a resource is managed using time restrictions.

The rest of this paper is organized as follows. The second section presents a semi-Markov model ${ }^{l l}$ of a stylized fishery and constructs two criteria for the manager of our fishery. The third section uses these criteria and discusses two approaches to the regulatory problem faced by a fishery manager. The fourth section concludes and offers suggestions for future research.

\section{SEASON LENGTH RESTRICTIONS IN A STOCHASTIC FISHERY}

\section{Preliminaries}

We use the theory of semi-Markov processes to characterize a fishery. In using a Markovian approach, we are following a suggestion contained in Perrings (1998) and followed by Batabyal and Beladi (2001), among others. Consider a stochastic process with states $0,1,2, \ldots$, that is now in state $i, i \geq 0$, and that has the following two properties. First, the probability that it will next enter state $j, j \geq 0$, is given by the transition probability $P_{i j}$. Second, given that the next state to be entered is $j$, the time until the transition from state $i$ to state $j$ is a random variable with the distribution function $G_{i j}(\cdot)$ with $G_{i j}^{c}(\cdot)=1-G_{i j}(\cdot)$. Now let $z(t)$ denote the state of the process at time $t$. Then $\{z(t): t \geq 0\}$ is a semi-Markov process.

Put differently, a semi-Markov process is a Markov chain with one salient difference. Whereas a Markov chain spends one unit of time in a state before making a transition to some other state, a semi-Markov process stays in a particular state for a random amount of time before making a transition to some other state. Let $J_{i}$ denote the distribution function of the time that $\{z(t)$ : $t \geq 0\}$ spends in state $i$ before making a transition and let $\sigma_{i}$ denote the expectation of this time in state $i$. Finally, let $T_{i i}$ be the time between successive transitions into state $i$ and let $\sigma_{i i}$ be the expectation of $T_{i i}$. In other words, $\sigma_{i i}=$ $E\left[T_{i i}\right]$. We are now in a position to discuss the characteristics of the fishery that is the subject of this paper.

\section{The Stochastic Fishery}

Consider a stochastic fishery-formally a semi-Markov process-that exists in one of three possible states. "State 1 is the healthy state of the fishery. In this state, fish are abundant and the fishery is open for fishing. State 2 is an intermediate state. In this state, the fish stock is lower than in state 1 but the total allowable catch (TAC) of fish has not yet been taken. Consequently, the fishery is still open for fishing. However, in state 2, the manager monitors the fish stock more carefully than in state 1 . State 3 is the state in which the fish species is endangered. In this state, the TAC has been taken and the fish stock is hazardously low. Consequently, if the manager determines that the fishery is in state 3 , then the fishing season is closed. Put differently, a season length restriction is now in place.

Let us formalize these observations. As a result of continued fishing, ecological/environmental factors (excessive predation, storms, unusually cold or warm water temperature), and human induced factors (water pollution), the fish stock in our fishery declines probabilistically. This means that the fishery stays in state 1 for a mean length of time $\sigma_{1}$ and then makes a transition either to state 2 with transition probability $P_{12}$, or to state 3 with transition probability $P_{13}$. If our fishery is in state 2 , then once again on account of the above mentioned reasons, the stock of fish declines probabilistically. This means that the fishery will stay in state 2 for a mean length of time $\sigma_{2}$ and then move to state 3 with transition probability $P_{23}$. When in state 3 , the fishing season is closed. Note that the length of time during which the fishing season is closed-to allow the fish stock to recover-is itself a random variable. Denote the mean length of the season closing by $\sigma_{3}$. Now depending on the length of this season closing, the fishery will return to either state 1 with transition probability $P_{31}$, or to state 2 with transition probability $P_{32}$. Let us now use these

\footnotetext{
"There are many references on semi-Markov processes. Excellent textbook accounts can be found in Medhi (1994, pp. 313-339) and in Ross (1996, pp. 213-218; 1997, pp. 379-381). Our primer on semi-Markov processes and this section's model are based on the discussion in Ross (1996, pp. 213218; 1997, pp. 379-381).

${ }^{\#}$ As Hartwick and Olewiler (1998. p. 153) have noted, fishery regulation, i.e. closing a fishing season, is a costly activity. Moreover, rarely does a manager have accurate information about all the possible stock values of a particular fish species. In other words, information about stock levels is typically incomplete and, on account of cost, a manager will generally regulate a fishery (close the fishing season) only when certain threshold stock levels have been reached. One possible threshold stock level is the one in which the TAC for the season has been taken. We model these features by truncating the state space of the fishery and by analyzing the resulting three state fishery. The reader should note that the model can easily be generalized to the case of a finite number of states.
} 
dynamic and stochastic attributes of this fishery and derive two criteria that our manager might optimize.

\section{Two Managerial Criteria}

\section{The First Criterion}

To derive the first managerial criterion, it will be necessary to compute the stationary probabilities for our three state fishery. Formally, we are interested in computing $P_{i}=\lim _{t \rightarrow \infty} \operatorname{Prob}\{z(t)=i / z(0)=j\}$ for any state $j$ and for states $i=1,2,3$. In words, given that our fishery is in state $j$ at time $t=0$, we want to compute the limiting probability, as time tends to infinity, that the fishery will be in state $i$. To perform this computation, let us denote the stationary probabilities of the embedded Markov chain of our fishery ${ }^{* *}$ by $\pi_{i}$, $i=1,2,3$. Now it is well known-see Eq. (7.23) in Ross (1997, p. 380)-that these limiting probabilities satisfy

$$
\pi_{j}=\sum_{i=1}^{i=3} \pi_{i} P_{i j}
$$

and

$$
\sum_{j=1}^{j=3} \pi_{j}=1
$$

Consequently, using the transition probabilities of the fishery and Eqs. (1) and (2), we can calculate the required limiting probabilities. These are

$$
\begin{gathered}
\pi_{1}=\frac{P_{31}}{1+P_{31}+P_{12} P_{31}+P_{32}}, \\
\pi_{2}=\frac{P_{12} P_{31}+P_{32}}{1+P_{31}+P_{12} P_{31}+P_{32}},
\end{gathered}
$$

and

$$
\pi_{3}=\frac{1}{1+P_{31}+P_{12} P_{31}+P_{32}} .
$$

To determine the stationary probabilities (denoted by $\left.P_{i}\right)$ of the fishery, we now use the Eq. (7.24) in Ross (1997, p. 380). This equation tells us that the $P_{i}^{\prime} s$ satisfy

$$
P_{i}=\frac{\pi_{i} \sigma_{i}}{\sum_{j=1}^{j=3} \pi_{j} \sigma_{j}}
$$

Equations (3)-(5) together give us the stationary probabilities that we are after. Performing the necessary mathematical computations, we get

$$
\begin{gathered}
P_{1}=\frac{P_{31} \sigma_{3}}{P_{31} \sigma_{1}+\left(P_{12} P_{31}+P_{32}\right) \sigma_{2}+\sigma_{3}} \\
P_{2}=\frac{\left(P_{12} P_{31}+P_{32}\right) \sigma_{2}}{P_{31} \sigma_{1}+\left(P_{12} P_{31}+P_{32}\right) \sigma_{2}+\sigma_{3}}
\end{gathered}
$$

and

$$
P_{3}=\frac{\sigma_{3}}{P_{31} \sigma_{1}+\left(P_{12} P_{31}+P_{32}\right) \sigma_{2}+\sigma_{3}} .
$$

In the context of our ecological-economic approach to the fishery management problem, these stationary probabilities (Eqs. (6) and (7)) have a distinct ecological meaning. As discussed in Krebs (1985, p. 587), Perrings (1998) and Batabyal (2000), these probabilities measure the asymptotic resilience of the fishery in each of these three states. Resilience is an ecological stability property and it refers to "the amount of disturbance that can be sustained [by an ecosystem] before a change in system control or structure occurs" Holling et al. (1995, p. 50). Put differently, we can think of the resilience of a fishery as a long run measure of the health of this fishery. Now if we rank the three states from this health perspective, then it should be clear to the reader that state 1 is the "healthiest" because the fish stock is abundant, state 2 is "healthy" because the fish stock is at an intermediate level, and state 3 is "unhealthy" because the fish stock is depleted. Using the words of Perrings (1998), state 1 is a "desirable" state and state 3 is an "undesirable" state.

As indicated earlier, the manager closes the fishing season if and only if the fishery is determined to be in state 3. Further, the mean length of this season closing is $\sigma_{3}$. With these two observations and the previous paragraph's discussion of the three states in mind, we can now state the first criterion for our fishery manager. Ideally, this manager would like to choose the expected length of the season closing $\sigma_{3}$ to maximize the long run probability of existing in the ecologically healthiest state 1 . Nevertheless, calculus and Eq. (6) tell us that $P_{1}$ is convex in $\sigma_{3}$. Therefore, it is not sensible to maximize $P_{1}$ over $\sigma_{3}$. As such, we presume that our manager selects $\sigma_{3}$ to minimize $P_{2}$, the long run probability of existing in the intermediate state 2 . Because the long run probability of existing in state 2 is the resilience of the fishery in state 2 , we can think of this minimization exercise as one that involves the simultaneous minimization of the fishery's resilience in the intermediate state. This is the ecological side of the management picture.

To see the economic side, note that fishery regulation brings benefits and costs to society. Season closings permit the fish stock to recover and thereby ensure that this fishery will continue to provide a flow of services to society in the future. These services may be consumptive (fish for food) or non-consumptive (recreational activities

\footnotetext{
${ }^{* *}$ For additional details on the embedded Markov chain of a semi-Markov process, see the references cited in footnote $\|$.
} 
such as boating and water sports). In contrast with such benefits, fishery regulation also imposes costs on society. Not only is it necessary to have an elaborate bureaucracy in place, but season closings lead to idle fishermen, unused capital, and equipment decay. Consequently, in deciding the length of the season closing, our manager must pay attention to both the benefits and the costs from regulation. In particular, because of political reasons, ${ }^{\dagger \dagger}$ we suppose that the fishery manager cannot let the net social benefit from regulation-which we model with the strictly decreasing and the strictly concave function $B\left(\sigma_{3}\right)$-fall below a certain acceptability threshold. Denote this threshold by $\hat{B}$. This discussion tells us that the economic side of the manager's problem is given by a constraint on the net benefit to society from the season closure. This constraint is $B\left(\sigma_{3}\right) \geq \hat{B}$.

To see why this net social benefit function is decreasing in the mean length of the season closing, recall that this function measures the economic benefit to society from the fishery's provision of consumptive and non-consumptive services. Now, when our fishery is in state 3 , a season length restriction is in place and the fishery is recovering. Therefore, during this time period, the fishery is not being used by society. This means that from a use perspective, the longer the average season length restriction, the lower the economic benefits. This is why the net social benefit function is decreasing in the length of the season closure restriction $\sigma_{3}$. This completes the derivation of the first managerial criterion and our discussion of the manager's optimization problem.

\section{The Second Criterion}

The second managerial criterion also involves working with a probability, but now the method of choosing the season length restriction and the focus of the manager are different. As in the previous section, once again we shall take a long run view of the fishery. Suppose that at time $t$ the fishery is in the undesirable state 3 . By choosing the season length restriction (now denoted by $\tau$ ), the manager can influence the state into which the fishery will next make a transition. Ideally, the manager would like this next state to be 1 . As such, if we let $X(t)$ be the time from $t$ until the next transition and $A(t)$ be the state entered at the first transition after $t$, then we can determine the long run probability of the fishery being in state 3 at time $t$, of making the next transition after time $t+\tau$, and of this next transition being into state 1 . In this instance, mathematically, we would want to compute $\lim _{t \rightarrow \infty} \operatorname{Prob}\{z(t)=$ $3, X(t)>\tau, A(t)=1\}$ and maximize this probability. However, it can be shown that this stationary probability is convex in $\tau$, therefore, it does not make much sense to maximize this probability. Given this state of affairs, we presume that our manager minimizes $\lim _{t \rightarrow \infty} \operatorname{Prob}\{z(t)=$ $3, X(t)>\tau, A(t)=2\}$, which is convex in $\tau$.
The reader will note that because of the non-concavity of $\lim _{t \rightarrow \infty} \operatorname{Prob}\{z(t)=3, X(t)>\tau, A(t)=1\}$ in $\tau$, our manager now attempts to indirectly increase the likelihood of moving the fishery to the "healthiest" state 1 by minimizing the probability of moving to state 2 from 3 . Moreover, unlike the probability derived in the previous sub-section, $\lim _{t \rightarrow \infty} \operatorname{Prob}\{z(t)=3, X(t)>\tau, A(t)=2\}$ is a joint probability. This stationary joint probability can be computed with the aid of Theorem 4.8.4 in Ross (1996, p. 217). Modifying this theorem to our problem, we get

$$
\begin{aligned}
\lim _{t \rightarrow \infty} \operatorname{Prob}\{z(t) & =3, X(t)=\tau, A(t)=2\} \\
& =P_{32} \frac{\int_{\tau}^{\infty} G_{32}^{c}(w) \mathrm{d} w}{\sigma_{33}} .
\end{aligned}
$$

The right hand side (RHS) of Eq. (8) is the product of two terms. The first term is the probability of making a transition from state 3 to 2 . The second term is the ratio of the integral of the tail distribution of the amount of time the fishery spends in state 3 before making a transition to state 2 to the expected amount of time between successive transitions into state 3. Following Perrings (1998) and Batabyal (2000), we can now give an ecological interpretation to this first term on the RHS of Eq. (8). Specifically, this term (probability) is the transient or the short run resilience of the fishery in the unhealthy state 3.

The second term on the RHS of Eq. (8) contains the manager's control variable, i.e. the season closure length $\tau$. The manager's objective is now to choose $\tau$ to minimize the probability on the left hand side (LHS) of Eq. (8). This is the ecological side of the management picture. The economic side is similar to that in the previous subsection. In deciding the length of the season closing, our manager pays attention to the benefits and the costs from regulation. As indicated previously, because of political reasons, the fishery manager will not let the net social benefit from regulation fall below a certain acceptability threshold. Consequently, the economic side of the manager's problem is given by the constraint $B(\tau) \geq \hat{B}$ where $B^{\prime}(\tau) \leq 0$ for reasons similar to those given in the previous sub-section. This completes the derivation of the second managerial criterion and our discussion of the manager's optimization problem. We now discuss the solutions to these two optimization problems and then we draw inferences for fisheries management.

\section{OPTIMAL FISHERY MANAGEMENT WITH ECOLOGICAL-ECONOMIC CRITERIA}

\section{Minimizing the Stationary Probability}

Recalling from the discussion in "The first criterion" section we see that the first problem faced by our manager involves the minimization of an ecological criterion

\footnotetext{
${ }^{\dagger \dagger}$ For more on the connections between political factors and fishery management see Karpoff (1987) and Hartwick and Olewiler (1998, pp. 152-175).
} 
subject to an economic constraint. The ecological criterion is the asymptotic resilience of the fishery in the middle-ofthe-road state 2 and the economic constraint tells us that the social net benefit from fishery management must exceed a benefit acceptability threshold. Formally, our manager solves

$$
\min _{\left\{\sigma_{3} \geq 0\right\}} \frac{\left(P_{12} P_{31}+P_{32}\right) \sigma_{2}}{P_{31} \sigma_{1}+\left(P_{12} P_{31}+P_{32}\right) \sigma_{2}+\sigma_{3}},
$$

subject to

$$
B\left(\sigma_{3}\right) \geq \hat{B} .
$$

Now, suppose that the solution to problem (9) and (10) yields an interior minimum at which the constraint binds. Then, omitting the complementary slackness conditions, the Kuhn-Tucker conditions for a minimum are

$$
\frac{-\left(P_{12} P_{31}+P_{32}\right) \sigma_{2}}{\left\{P_{31} \sigma_{1}+\left(P_{12} P_{31}+P_{32}\right) \sigma_{2}+\sigma_{3}\right\}^{2}}=\lambda B^{\prime}\left(\sigma_{3}\right)
$$

where $\lambda$ is the multiplier on the social net benefit constraint (Eq. (10)) and

$$
B\left(\sigma_{3}\right)=\hat{B} .
$$

On solving Eqs. (11) and (12) in unison, we get the optimal values of the season closure length $\left(\sigma_{3}^{*}\right)$ and the shadow value of the social net benefit constraint $\left(\lambda^{*}\right)$. The principle condition here is Eq. (11). This equation tells us that in choosing the season length restriction optimally, the manager will balance ecological and economic considerations. Specifically, $\sigma_{3}^{*}$ will be chosen so that the marginal effect of the season length restriction on the asymptotic resilience of the fishery in state 2 (the LHS) is equal to the product of the shadow value of the social net benefit constraint and the marginal benefit to society from choosing the season closure length optimally (the RHS).

If the season closure length is chosen in this way, then we can be reasonably sure that the fishery will be robust in the long run. From an ecological perspective, this means that the resilience of this fishery in the middle-of-the-road state 2 will be low. In economic terms, this means that the fishery will provide society with a flow of consumptive and non-consumptive services.

\section{Minimizing the Stationary Joint Probability}

Recalling from "The second criterion" section we see that in this version of the management problem, the manager's primary objective is to choose the season closure length so that the long run probability of the fishery being in state 3 at time $t$, of making the next transition after time $t+\tau$, and of this next transition being into state 2 , is small. Formally, the manager solves

$$
\min _{\{\tau \geq 0\}} P_{32} \frac{\int_{\tau}^{\infty} G_{32}^{c}(w) \mathrm{d} w}{\sigma_{33}},
$$

subject to

$$
B(\tau) \geq \hat{B}
$$

As stated, this minimization problem is ungainly. Consequently, to illustrate our approach, we suppose that the amount of time the fishery spends in state 3 before making a transition to state 2 is exponentially distributed. Then, integrating the tail distribution function for an exponentially distributed random variable-see Jeffrey (1995, p. 163 and 248) — and then evaluating this integral at the upper and the lower limits, we get

$$
\int_{\tau}^{\infty} G_{32}^{c}(w) \mathrm{d} w=\frac{1}{\alpha} \exp \{-\alpha \tau\}
$$

where $\alpha$ is the parameter of the exponential distribution function. Using Eq. (15), our manager's minimization problem becomes

$$
\min _{\{\tau \geq 0\}} P_{32} \frac{\frac{1}{\alpha} \exp \{-\alpha \tau\}}{\sigma_{33}}
$$

subject to Eq. (14). Now, as in the previous sub-section, suppose that the solution to problem (14)-(16) yields an interior minimum at which the constraint binds. Then, excluding the complementary slackness conditions, the Kuhn-Tucker conditions for a minimum are

$$
\frac{P_{32}}{\sigma_{33}}[-\exp \{-\alpha \tau\}]=\theta B^{\prime}(\tau),
$$

where $\theta$ is the multiplier on the constraint (Eq. (14)) and

$$
B(\tau)=\hat{B}
$$

On solving Eqs. (17) and (18) together, we obtain the optimal values of the season closure length $\left(\tau^{*}\right)$ and the shadow price of the social net benefit constraint $\left(\theta^{*}\right)$. The "ecological-economic" optimality condition is Eq. (17). This equation tells us that when the manager's primary concern is to minimize the long run probability of moving to the intermediate state 2 from the undesirable state 3 , (s)he will select the length of the season closure so that the marginal effect of the season closure on the long run probability of the fishery being in state 3 at time $t$, of making the next transition after time $t+\tau$, and of this next transition being into state 2 (the LHS) is equal to the product of the shadow value of the social net benefit constraint and the marginal net benefit to society from selecting the season closure length optimally (the RHS).

It $\tau^{*}$ is chosen in this way, then we can be fairly sure that our fishery will be "sustainable" in the long run. The reader will note that in the context of this paper, sustainability has a twofold meaning. From an ecological standpoint, sustainable means that the fishery will not be 
resilient in the undesirable state 3 . In economic terms, sustainable means that the fishery will be able to provide society with a flow of consumptive and non-consumptive services over time.

It is our conjecture that a conservative fishery manager will want to solve the minimization problem of this subsection rather than the minimization problem of the "Minimizing the stationary probability" section. This is because in the optimization problem of this sub-section, the focus is explicitly on minimizing the long run probability of the fishery moving to state 2 from state 3 . In contrast to this, in the optimization problem of the "Minimizing the stationary probability" section, the focus is simply on minimizing the long run proportion of time that the fishery spends in the middle-of-the-road state 2 . In this connection, one can ask the following question: Which problem solution results in a longer season closure? In other words, is $\tau^{*}>\sigma_{3}^{*}$ or is $\tau^{*} \leq \sigma_{3}^{*}$ ? The reader should note that without significant additional structure on the two problems that we have been analyzing, this question cannot be resolved definitively.

\section{CONCLUSIONS}

We accomplished three tasks in this paper. First, in the "Season length restrictions in a stochastic fishery" section, we used the theory of semi-Markov processes to provide an ecological-economic model of a stylized fishery that is managed with season length restrictions. Next, we used the dynamic and the stochastic properties of this fishery to derive two managerial criteria that are meaningful from an ecological standpoint. We stress that because these two criteria are probabilities, our approach to the fishery management problem is very different from traditional economic approaches to this problem. Finally, in the "Optimal fishery management with ecological-economic criteria" section, we used these two criteria to study two fishery management problems from an ecologicaleconomic perspective. In this perspective, the principal focus of the manager is on selecting the season closure length to minimize the amount of time the fishery spends in the middle-of-the-road state 2 .

The analysis contained in this paper can be extended in a number of directions. In what follows, we suggest two possible extensions. First, recall that in "The second criterion" section, our manager's objective was to minimize the stationary probability of the fishery being in state 3 at time $t$, of making the next transition after time $t+\tau$, and of this next transition being into state 2 . This approach does not require the manager to condition this probability on any known information. Consequently, it would be useful to compare the solution to the manage- ment problem when this criterion is used with the solution of a management problem in which the manager minimizes the stationary conditional probability that the next state visited after time $t$ is 2 , given that the fishery is in state 3 at time $t$.

Second, we modeled the fishery as a semi-Markov process. Although this is a fairly general stochastic process, it is still Markovian in the sense that a semiMarkov process changes states in accordance with a Markov chain. Consequently, it would be useful to know whether any aspects of our analysis change when a more general stochastic process is used to model the fishery. Studies of fisheries management that incorporate these aspects of the problem into the analysis will provide additional insights into the management of fisheries whose behavior is governed by a great deal of uncertainty.

\section{Acknowledgements}

The authors acknowledge financial support from the Utah Agricultural Experiment Station, Utah State University, Logan, UT 84322-4810, by way of project UTA 024. The usual disclaimer applies.

\section{References}

Batabyal, A.A. (2000) "Quantifying the Transient Response of Ecological-Economic Systems to Perturbations", Environmental Impact Assessment Review, 20, 125-133.

Batabyal, A.A. and Beladi, H. (2001) Time Restrictions in Natural Resource Management: A Dynamic and Stochastic Analysis (Rochester Institute of Technology, Unpublished manuscript.

Gulland, J.A., (1988) In: Fish Population Dynamics, 2nd Ed. (Wiley, Chichester, England).

Hartwick, J.M. and Olewiler, N.D. (1998) The Economics of Natural Resource Use, 2nd Ed. (Addison-Wesley, Reading, MA).

Holling, C.S., Schindler, D.W., Walker, B. and Roughgarden, J. (1995) "Biodiversity in the functioning of ecosystems: an ecological synthesis", In: Perrings, C., Maler, K.G., Folke, C., Holling, C.S. and Jansson, B.O., eds, Biodiversity Loss (Cambridge University Press, Cambridge, England).

Homans, F.R. and Wilen, J.E. (1997) "A model of regulated open access resource use", Journal of Environmental Economics and Management 32, 1-21.

Jeffrey, A. (1995) Handbook of Mathematical Formulas and Integrals (Academic Press, San Diego, CA).

Karpoff, J.M. (1985) "Time, capital intensity, and the cost of fishing effort", Western Journal of Agricultural Economics 10, 254-258.

Karpoff, J.M. (1987) "Suboptimal controls in common resource management: the case of the fishery", Journal of Political Economy 95, 179-194.

Krebs, C.J. (1985) Ecology, 3rd Ed. (Harper and Row Publishers, New York).

Matulich, S.C., Mittelhammer, R.C. and Roberter, C. (1996) "Toward a more complete model of individual transferable fishing quotas: implications of incorporating the processing sector", Journal of Environmental Economics and Management 31, 112-128.

Medhi, J. (1994) Stochastic Processes, 2nd Ed. (Wiley, New Delhi, India).

Perrings, C. (1998) "Resilience in the dynamics of economy-environment systems", Environmental and Resource Economics 11, 503-520.

\footnotetext{
${ }^{*}$ The stationary probability of existing in state 2 can also be interpreted as the long run proportion of time that the fishery spends in state 2 . For more on this, see Corollary 4.8.2 in Ross (1996, p. 214).
} 
Richards, R.A. and Rago, P.J. (1999) "A case history of effective fishery management: Chesapeake Bay striped bass", North American Journal of Fisheries Management 19, 356-375.

Ross, S.M. (1996) Stochastic Processes, 2nd Ed. (Wiley, New York, NY).

Ross, S.M. (1997) Introduction to Probability Models, 6th Ed. (Academic Press, San Diego, CA).
Weninger, Q. and Strand, I.E. (1998) Regulating Production Time to Control Output: An Application to a Harvest Technology (Utah State University, Unpublished manuscript.

Williams, J.G. (1999) "Stock dynamics and adaptive management of habitat: an evaluation based on simulations", North American Journal of Fisheries Management 19, 329-341. 


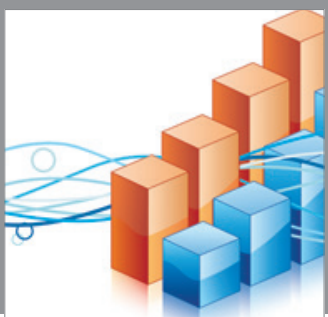

Advances in

Operations Research

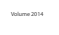

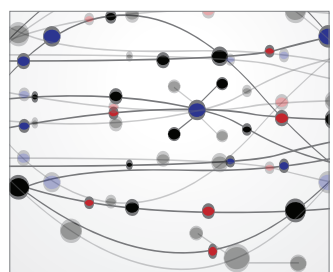

\section{The Scientific} World Journal
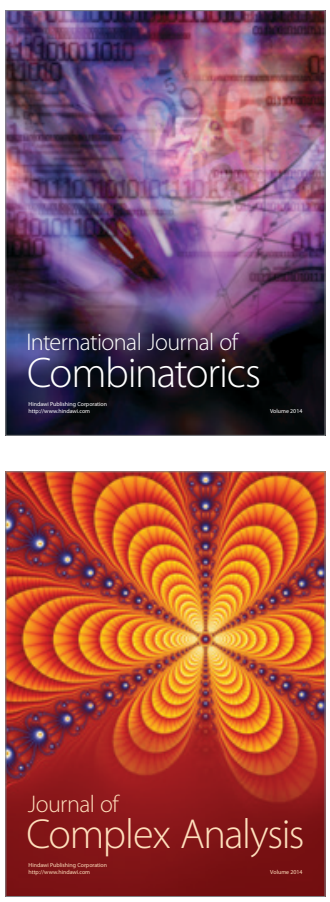

International Journal of

Mathematics and

Mathematical

Sciences
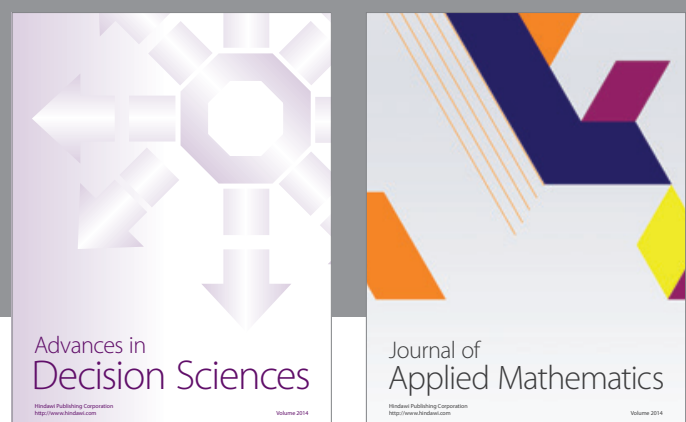

Journal of

Applied Mathematics
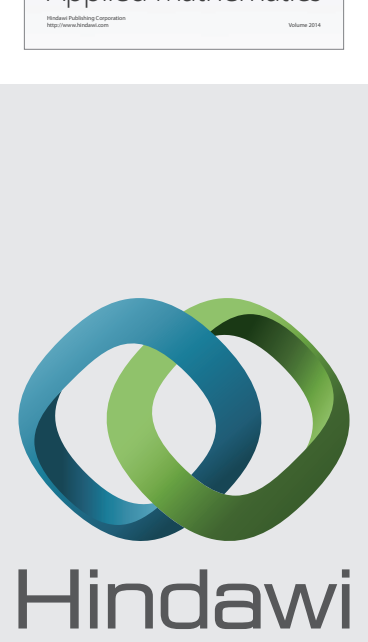

Submit your manuscripts at http://www.hindawi.com
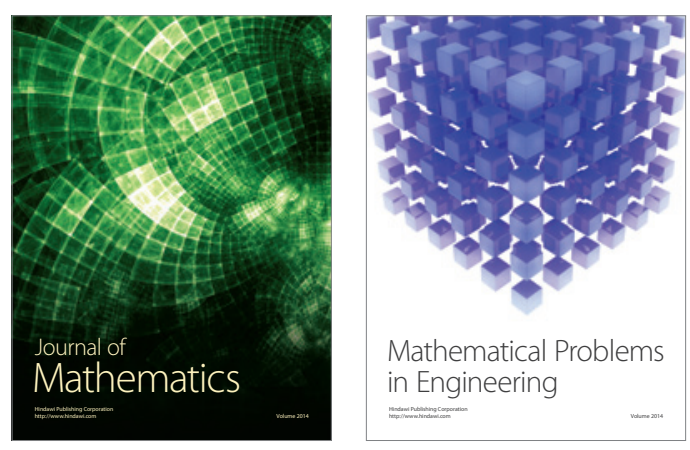

Mathematical Problems in Engineering
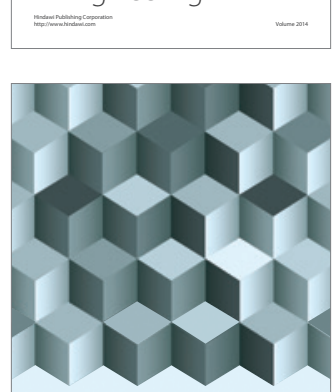

Journal of

Function Spaces
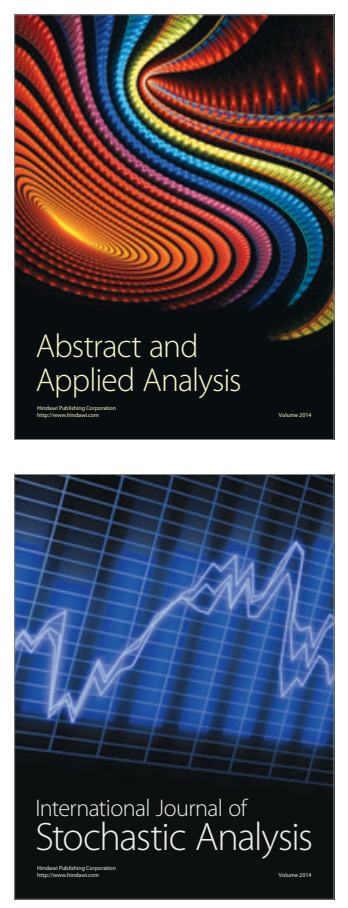

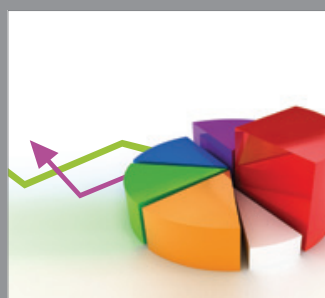

ournal of

Probability and Statistics

Promensencen
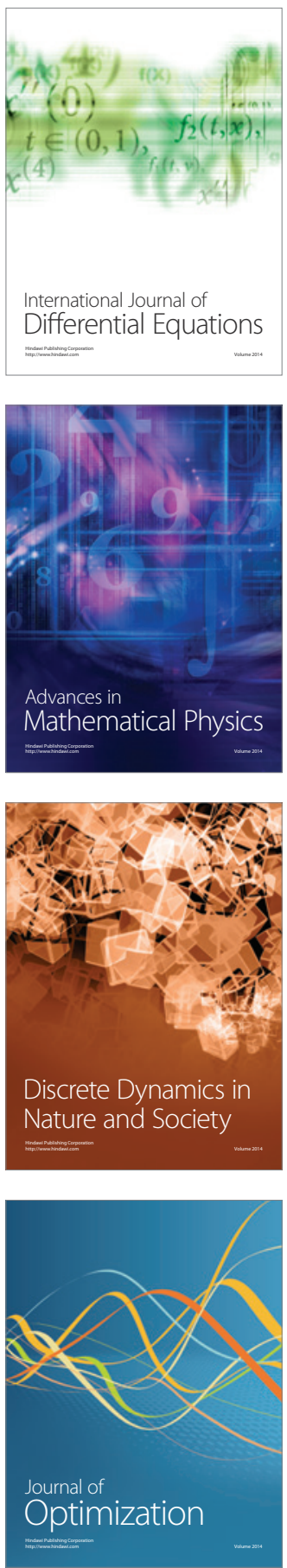